\title{
Simulating Wrinkles in Facial Expressions on an Anatomy-Based Face
}

\author{
Yu Zhang, Terence Sim, and Chew Lim Tan \\ School of Computing, National University of Singapore, Singapore 117543
}

\begin{abstract}
This paper presents a muscle-driven wrinkle model for simulating dynamic wrinkles that appear during facial expressions, which is geometric in nature. Wrinkles are generated on an anatomy-based face model that incorporates a structure of skin, muscles and skull for physically-based animation. Corresponding to two types of facial muscles, a geometric model is developed to govern how the wrinkle amplitude evolves locally upon skin deformation, taking into account the properties of real wrinkles. During facial animation, wrinkles are generated in the local regions influenced by muscle contraction, simulating resistance to compression of tissues. Using our method, realistic wrinkles can be dynamically rendered on the adaptively refined face mesh at interactive rates.
\end{abstract}

\section{Introduction}

Facial expressions exhibit not only facial feature motions, but also subtle changes in skin appearance such as facial creases and wrinkles. These details, or expressive wrinkles, constitute major elements affecting the visual appearance of the face. Therefore, realistic modeling of expressive wrinkles contributes significantly to the overall realism of facial animation.

One class of methods to generate facial wrinkles is the texture mapping approaches such as bump texture mapping and color texture mapping [2, 5, 6]. Although being efficient and giving acceptable visual results in simulating facial wrinkles formed due to skin texture and age, this technique has some inherent limitations. First, it is difficult to mimic the wrinkle dynamics during animation. Second, as the skin geometry remains undeformed, the perceptible geometrical and structural changes can not be modeled.

Another class of techniques, known as physically-based methods [3, 7, 11, 12], attempt to model the dynamic wrinkling as the result of skin deformation by approximating the biomechanical properties of real skin. Results of the physically-based methods are realistic, but the process is not real-time due to expensive computation of the underlying mass-spring or finite element system.

Geometric methods solve the difficulties associated with two classes of methods mentioned above by simulating the perceptible geometrical deformation of skin for rendering at a high simulation speed [1, 4, 8, 9]. Although these methods are efficient to produce convincing results, they reveal the difficulties in intuitive control such as adjusting size and position of wrinkles and involve painstaking tasks such as manually drawing wrinkle patterns in textures. Since expressive wrinkles are formed due to compression of the skin surface as a result of muscle contraction, our goal is to easily model wrinkles according to the influence of virtual facial muscles. 
In this paper, we present a muscle-driven wrinkle model from an anatomic point of view for efficiently simulating expressive wrinkles. Our method generates wrinkles on an anatomy-based face model. Corresponding to two types of facial muscles, a geometric model is developed to govern how the wrinkle amplitude evolves locally upon skin deformation, taking into account the properties of real wrinkles. During facial animation, wrinkles are generated in the local regions influenced by muscle contraction, simulating resistance to compression of tissues. Our method simulates wrinkles that can be dynamically rendered on the adaptively refined face mesh at interactive rates.

\section{Muscle-Driven Modeling of Wrinkles}

\subsection{An Anatomy-Based Face Model}

We have developed an anatomy-based face model for physically-based facial animation [13]. The model encapsulates three structural layers: skin, muscles, and skull (see Fig. 1). The reader is referred to [13] for technical details.

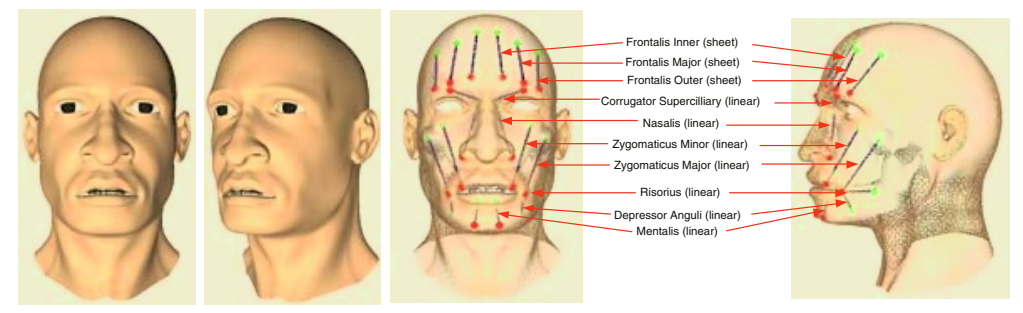

Fig. 1. The anatomy-based face model. Left: face geometry. Right: multi-layer anatomic structure of the skin, muscles and skull

Since expressive wrinkles are dynamically generated due to shrinking of the skin surface as a result of muscle contraction, we propose a muscle-driven wrinkle model to automatically simulate them. In our face model, we use two kinds of physicallybased facial muscle models that are based on Waters' muscle model [10]: the linear muscle for the majority of facial muscles, the sheet muscle for the forehead muscles (Frontalis).

\subsection{Modeling of Wrinkles Driven by the Linear Muscle}

Muscle Model and Its Wrinkling Region. A linear muscle is defined by an insertion point, the end of the muscle attached to skin; an attachment point, the end of the muscle fixed to bone; and the area of influence (AOI), the portion of the surface affected by the muscle contraction. The effect of facial muscle contraction is to pull the facial skin from the area of the muscle insertion point to the static muscle attachment point on the bone. Fig. 2 2 illustrates the linear muscle model and its AOI with the following definitions: 
$\mathbf{x}_{i}:$ arbitrary facial skin point

$\mathbf{f}_{i}$ : muscle force applied to $\mathbf{x}_{i}$

$\mathbf{m}_{A}$ : attachment point of linear muscle at the skull

$\mathbf{m}_{I}$ : insertion point of linear muscle at the facial skin

$\phi$ : maximal angle of muscle influence

$\varphi_{i}$ : angle between the central muscle fiber $\mathbf{m}_{A} \mathbf{m}_{I}$ and $\mathbf{m}_{A} \mathbf{x}_{i}$

$R$ : maximal radius of influence

$l_{i} \quad$ : distance between $\mathbf{x}_{i}$ and $\mathbf{m}_{A}$ projected onto the central muscle fiber $\mathbf{m}_{A} \mathbf{m}_{I}$

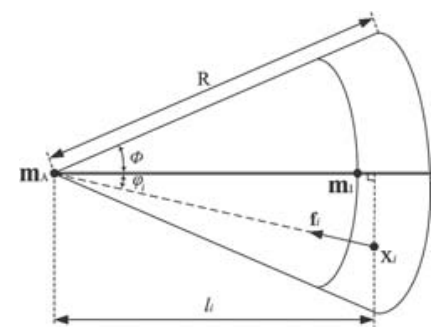

Fig. 2. Linear muscle model

For an arbitrary vertex $\mathbf{x}_{i}$ on the skin mesh, its distance from the muscle attachment point projected onto the central muscle fiber $\mathbf{m}_{A} \mathbf{m}_{I}$ is calculated as:

$$
l_{i}=\left\|\mathbf{m}_{A}-\mathbf{x}_{i}\right\| \cos \left(\varphi_{i}\right)
$$

where $\|\cdot\|$ denotes the Euclidean norm. $l_{i}$ and $\varphi_{i}$ weight the influence of the linear muscle at vertex $\mathbf{x}_{i}$ separately for length factor $\lambda_{i}$ and angular factor $\gamma_{i}$ :

$$
\lambda_{i}=\frac{l_{i}}{R}, \quad \gamma_{i}=\frac{\varphi_{i}}{\phi}
$$

$\lambda_{i}$ defines the normalized longitudinal distance of vertex $\mathbf{x}_{i}$ to the muscle attachment point. $\gamma_{i}$ is defined in $[0,1]$ and represents the distance between $\mathbf{x}_{i}$ and the central muscle fiber in the angular direction. The wrinkling region of the influenced skin surface is a sub-area of muscle's AOI. The set of vertices inside the wrinkling region is

$$
S_{w}=\left\{\mathbf{x}_{i} \mid \lambda_{w} \leq \lambda_{i} \leq 1 \text { and } 0 \leq \gamma_{i} \leq 1\right\}
$$

where $\lambda_{w}$ is a user-specified factor defined in $[0,1]$ to control the size of the wrinkling region. The smaller $\lambda_{w}$, the larger the wrinkling region.

Geometric Wrinkle Model. The wrinkle amplitude is calculated for all vertices that are originally (before activating the muscle contraction) inside the wrinkling region. The wrinkle amplitude at a vertex $\mathbf{x}_{i}$ is given as wrinkle amplitude function $W\left(\mathbf{x}_{i}\right)$ :

$$
W\left(\mathbf{x}_{i}\right)=H\left(w_{i}\right) A\left(\varepsilon_{i}\right) A t\left(\varphi_{i}\right)
$$

where $H\left(w_{i}\right)$ is the shape function used to obtain natural wrinkle shape, anisotropic function $A\left(\varepsilon_{i}\right)$ modulates the wrinkle amplitude to model irregularity of wrinkles, and $A t\left(\varphi_{i}\right)$ is the attenuation function. 


\section{Wrinkle Shape}

The cross sectional shape of the wrinkle is defined by the shape function $H\left(w_{i}\right)$, a height function of $3 \mathrm{D}$ position of the vertex:

$$
H\left(w_{i}\right)=\left\{\begin{array}{l}
k_{0}\left(1+\left(\frac{w_{i}-u}{u}\right)^{2}\right)^{\alpha}\left(\frac{w_{i}-u}{u}\right)+d \quad 0 \leq w_{i} \leq u \\
-k_{0}\left(1+\left(\frac{w_{i}-u}{u}\right)^{2}\right)^{\alpha}\left(\frac{w_{i}-u}{u}\right)+d u<w_{i} \leq 2 u
\end{array}\right.
$$

The $H\left(w_{i}\right)$ curve depicted in Fig. 3 illustrates how wrinkling amplitude evolves. In Eq. 5 . $u$ is the half cycling length. Given a predefined value to the number of wrinkles $\left(n_{w}\right)$ generated by the contraction of the muscle, $u$ is obtained as:

In Eq. 5

$$
u=\frac{\left(1-\lambda_{w}\right) R}{2 n_{w}}
$$

$$
w_{i}=l_{i}-\lambda_{w} R-\operatorname{int}\left(\frac{l_{i}-\lambda_{w} R}{2 u}\right) 2 u
$$

As int $(\cdot)$ truncates a real number to the biggest integer number that is smaller than it, Eq.7periodically maps $l_{i}$ into $[0,2 u]$ with a frequency of $n_{w}$.

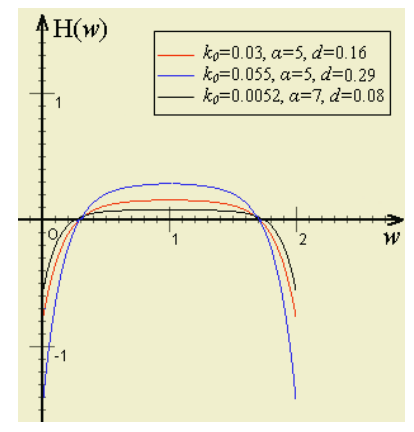

Fig. 3. The shape function with different parameter values $(u=1.0)$

Three intuitive parameters are introduced in the shape function to control wrinkle characteristics. The parameter $k_{0}$ controls the depth of the furrow in a wrinkle and hence the name furrow depth factor, $\alpha$ is the shape modulation factor that controls the modulation of the wrinkle shape, and $d$ is the bulge height parameter that defines the maximal height of the bulge in a wrinkle. By adjusting them, shape function $H\left(w_{i}\right)$ can be chosen to simulate the real wrinkle property: the furrow of a wrinkle is sharp and the bulge is round. Moreover, the shape function should satisfy the following equation:

$$
\int_{0}^{2 u} H(w) d w=0
$$

This means that the volume depressed at the furrow goes to the side to make up the bugle, which simulates the incompressibility of skin. Using Eq. 8, we can express the bulge height parameter $d$ as a function of $k_{0}$ and $\alpha$, which reduces the parameter tuning. 


\section{Modulation of Wrinkle Amplitude}

Obviously, expressive wrinkles do not all react in the same way to skin deformation, varying according to their alignment with the direction of skin compression. An anisotropic function $A\left(\varepsilon_{i}\right)$ is introduced, which modulates wrinkle amplitude according to skin surface deformation. Suppose a vertex $\mathbf{x}_{i}$ is connected to one of its neighbors $\mathbf{x}_{j}$ by an edge with the rest length $L_{i j}$ (see irg. 4). During animation, we compute at each time step the edge shrinkage $\tau_{i j}$ for the edge $\mathbf{x}_{i} \mathbf{x}_{j}$ :

$$
\tau_{i j}=\frac{L_{i j}-\left\|\mathbf{x}_{i}-\mathbf{x}_{j}\right\|}{L_{i j}}
$$

$\tau_{i j}$ is the amount of shortening relative to the original length, which equals 0 in the rest state. The skin is deformed in the direction of muscle contraction and wrinkles become more pronounced when the skin surface shrinks perpendicularly to their directions. Therefore, the more the direction of an edge runs parallel to the direction of muscle contraction, the more the wrinkle amplitude will react to variation in that edge's length. The compression rate $\varepsilon_{i}$ at the vertex $\mathbf{x}_{i}$ is defined as:

$$
\varepsilon_{i}=\frac{\sum_{j \in \Omega_{i}} \tau_{i j} \cos \left(\theta_{i j}\right)}{\sum_{j \in \Omega_{i}} \cos \left(\theta_{i j}\right)}
$$

where $\Omega_{i}$ denotes the set regrouping all neighboring vertices that are linked to $\mathbf{x}_{i}, \theta_{i j}$ is the angle between edge $\mathbf{x}_{i} \mathbf{x}_{j}$ and the direction of muscle contraction $\mathbf{x}_{i} \mathbf{m}_{A}$ (see Fig. 4). The weight $\cos \left(\theta_{i j}\right)$ measures the direction similarity of the considered edge to the muscle contraction direction.

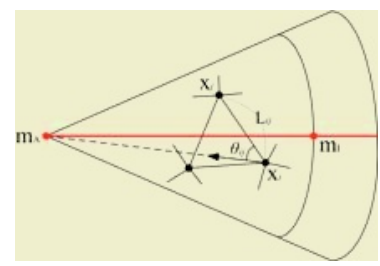

Fig. 4. Shorting of an edge contributes to the compression of the skin surface at a vertex

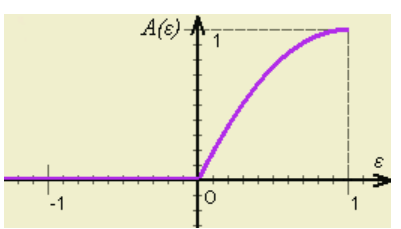

Fig. 5. Plot of the anisotropic function

The wrinkle amplitude varies from maximal amplitude for maximal compression $\left(\varepsilon_{i}=1\right)$ to null for expansion. Negative value of $\varepsilon_{i}$ means that stretching tends to make the wrinkles disappear. We model it by defining the following anisotropic function:

$$
A\left(\varepsilon_{i}\right)= \begin{cases}\cos \left(\frac{\pi}{2}\left(1-\varepsilon_{i}\right)\right) & 0 \leq \varepsilon_{i} \leq 1 \\ 0 & \varepsilon_{i}<0\end{cases}
$$

The function curve is depicted in Fig. 5 


\section{Attenuation of Wrinkle Amplitude}

The wrinkle amplitude is also scaled by the attenuation function $\operatorname{At}\left(\varphi_{i}\right)$ :

$$
A t\left(\varphi_{i}\right)=\left\{\begin{array}{lr}
1 & 0 \leq \varphi_{i} \leq \varphi^{c} \\
-\frac{1}{2}\left(1+\left(\frac{\varphi_{i}-\varphi^{c}}{\phi-\varphi^{c}}\right)^{2}\right)\left(\frac{\varphi_{i}-\varphi^{c}}{\phi-\varphi^{c}}\right)+1 \varphi^{c}<\varphi_{i} \leq \phi
\end{array}\right.
$$

where $\varphi_{i}$ is the angular distance of a vertex from the central muscle fiber $\mathbf{m}_{A} \mathbf{m}_{I}$. At $\left(\varphi_{i}\right)$ defines an attenuation profile which dictates the way the wrinkle height decreases and then vanishes when wrinkles go away from the central muscle fiber. Parameter $\varphi^{c}$ controls the angular distance from the central muscle fiber where the wrinkle amplitude begins to decrease (see Fig. 6(a)). Fig. 6(b) shows the graph of $A t\left(\varphi_{i}\right)$.

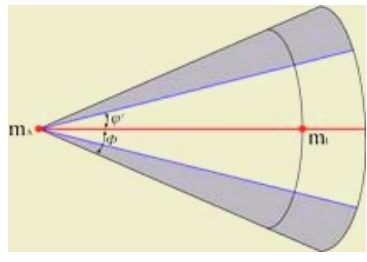

(a)

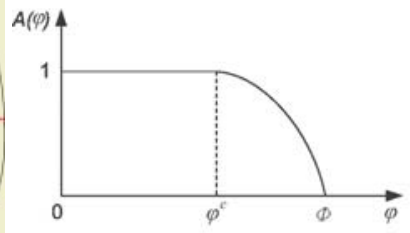

(b)

Fig. 6. (a) Attenuation area (gray area) in the wrinkling region of the linear muscle. (b) The attenuation function

\subsection{Modeling of Wrinkles Driven by the Sheet Muscle}

Muscle Model and Its Wrinkling Region. A sheet muscle is a series of almostparallel fibers spread over a rectangular area and it contracts to a group of separated muscle fiber nodes. The obvious example is the Frontalis, which lies on the forehead. Fig.77 illustrates the AOI of the sheet muscle model.

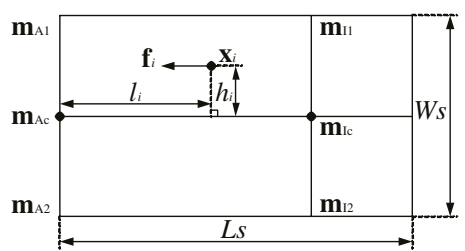

Fig. 7. Sheet muscle model

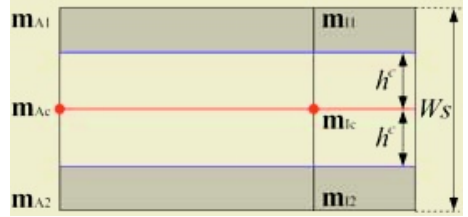

Fig. 8. Attenuation area (gray area) in the wrinkling region of the sheet muscle

Two points $\mathbf{m}_{A 1}$ and $\mathbf{m}_{A 2}$ specify the attachment line of the sheet muscle. $\mathbf{m}_{A c}$ is the middle point of the attachment line. Similarly, the points $\mathbf{m}_{I 1}$ and $\mathbf{m}_{I 2}$ specify the insertion line of the sheet muscle, and $\mathbf{m}_{I c}$ is the middle point of the insertion line. Vector $\mathbf{m}_{A c} \mathbf{m}_{I c}$ is the central muscle fiber. The denotations of other parameters are

$$
\begin{array}{ll}
\mathbf{x}_{i} & : \text { arbitrary facial skin point } \\
\mathbf{f}_{i} & : \text { muscle force applied to } \mathbf{x}_{i}
\end{array}
$$


$L s$ and $W s$ : length and width of sheet muscle's AOI, respectively

$l_{i} \quad: \quad$ distance between $\mathbf{x}_{i}$ and the muscle attachment line

$h_{i} \quad:$ distance between $\mathbf{x}_{i}$ and the central muscle fiber

Similar to the linear muscle model, the length factor $\lambda_{i}$ and width factor $\gamma_{i}$ associated with a vertex $\mathbf{x}_{i}$ are defined as:

$$
\lambda_{i}=\frac{l_{i}}{L s}, \quad \gamma_{i}=\frac{2 h_{i}}{W s}
$$

The wrinkling region is a sub-area of sheet muscle's AOI. The set of vertices inside the wrinkling region is

$$
S_{w}=\left\{\mathbf{x}_{i} \mid \lambda_{w} \leq \lambda_{i} \leq 1 \quad \text { and } \quad 0 \leq \gamma_{i} \leq 1\right\}
$$

where $\lambda_{w}$ in the range $[0,1]$ controls the size of the wrinkling region.

Geometric Wrinkle Model. The wrinkle amplitude function defined in Eq. 4 is employed for wrinkle generation, after modification of a few parameters. For the shape function $H\left(w_{i}\right)$, parameter $R$ in Eq. 6 and 7 is substituted by $L s$, the length of sheet muscle's AOI. For the anisotropic function $A\left(\varepsilon_{i}\right)$, since the direction of muscle contraction is parallel to the central muscle fiber $\mathbf{m}_{A c} \mathbf{m}_{I c}, \theta_{i j}$ in Eq.10 becomes the angle between the mesh edge and $\mathbf{m}_{A c} \mathbf{m}_{I c}$. The attenuation function is modified as:

$$
A t\left(h_{i}\right)=\left\{\begin{array}{lc}
1 & 0 \leq h_{i} \leq h^{c} \\
-\frac{1}{2}\left(1+\left(\frac{h_{i}-h^{c}}{W s / 2-h^{c}}\right)^{2}\right)\left(\frac{h_{i}-h^{c}}{W s / 2-h^{c}}\right)+1 & h^{c}<h_{i} \leq W s / 2
\end{array}\right.
$$

where $h_{i}$ is the distance between the vertex and the central muscle fiber, $h^{c}$ controls the starting position of attenuation (see Fig. (8).

\section{Experimental Results}

Fig. 9 shows examples of some facial expressions simulated with and without wrinkles, and compares them with the actual ones. Each expression is generated by activating a group of muscles:

- Surprise: Contractions of pairs of Frontalis Major, Frontalis Inner, and Frontalis Outer (sheet muscles) raise the eyebrows and cause wrinkles on the forehead.

- Frowning: Contractions of the pair of Corrugator Supercilliary (linear muscle) result in frowning of the eyebrows and large-scale wrinkles between the eyebrows.

- Fear: Frontalis Inner and Corrugator Supercilliary are activated to raise the inner portion of eyebrows and to stretch eyebrows towards the face center, respectively.

Fig. 10 shows the snapshots of dynamic wrinkle generation within different expressions. Results reveal that the generated expressive wrinkles satisfactorily enhance the realism of facial animation, making the simulated expressions more convincing. 


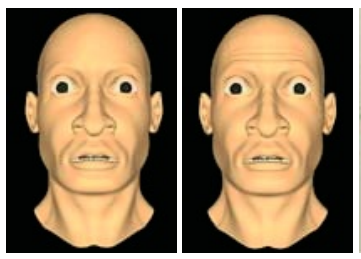

(a) Surprise
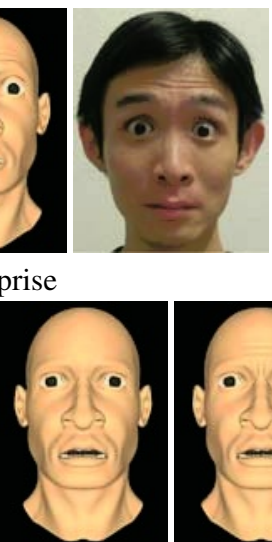
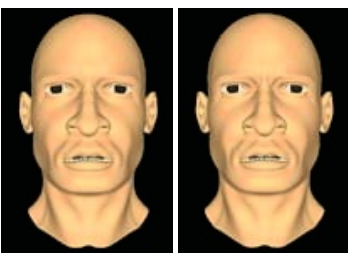

(b) Frowning
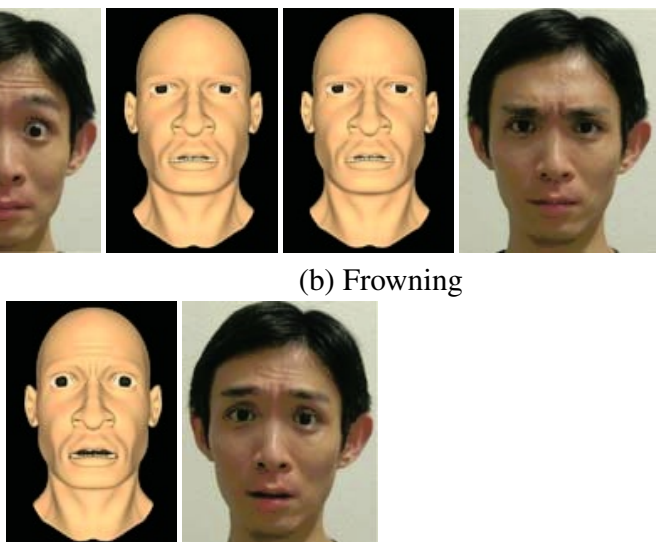

(c) Fear

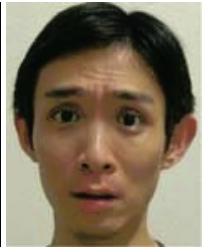

Fig. 9. Facial expressions simulated on the face model. Left to right: expression without wrinkles, expression with wrinkles, actual expression generated by a subject (each example)

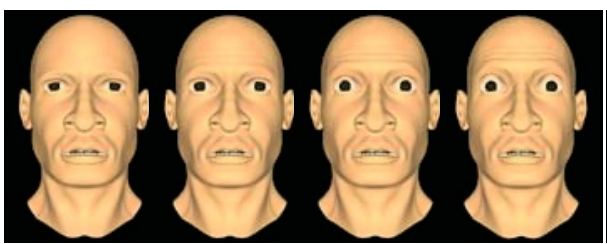

(a) Surprise

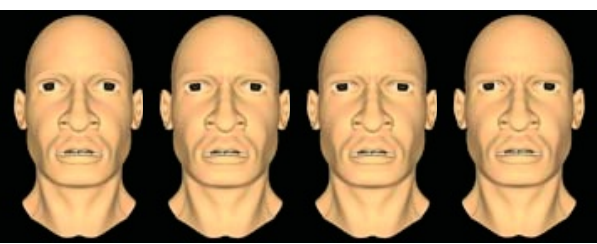

(b) Frowning

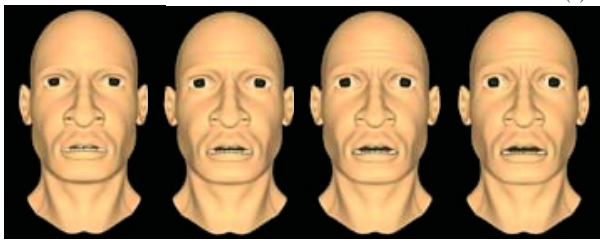

(c) Fear

Fig. 10. Dynamic simulation of the expressive wrinkles

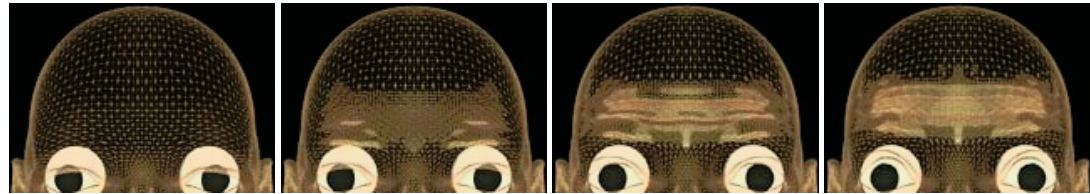

Fig. 11. Adaptive refinement of the face mesh in dynamic wrinkle simulation

The original face mesh consists of about 4,500 triangles. In order to correctly capture the wrinkle shapes, we implement our wrinkling process using a dynamically adaptive refinement scheme of the original mesh. The adaptive refinement is based on a recursive 
quaternary subdivision that is applied on the fly where surface curvature (measured from the vertex surface normals) of influenced triangles is too large. Fig. 11 shows a mesh detail of the deformed forehead region during simulation of surprise.

\section{Conclusion}

This paper has presented a geometric muscle-driven model for simulation of expressive wrinkles. Expressive wrinkles are automatically defined according to facial muscle anatomy. A geometric model governs evolution of wrinkle amplitude in the local deformed regions, taking into account the properties of real wrinkles. It provides intuitive parameters for easy control over wrinkle characteristics and is adapted to two types of facial muscles. Thanks to its geometric nature, our method simulates wrinkles that can be dynamically rendered at interactive rates.

For future work we will introduce a collision detection procedure in our implementation to prevent neighboring folds from intersecting. We would also like to develop the wrinkle model corresponding to the sphincter muscles that surround the eyes and mouth for simulating more realistic and complex deformations.

\section{References}

1. Y. Bando, T. Kuratate, and T. Nishita. "A simple method for modeling wrinkles on human skin", Proc. Pacific Graphics 2002, pp. 166-175, 2002.

2. J. Blinn. "Simulation of wrinkled surface", Proc. SIGGRAPH'78, pp. 286-292, 1978.

3. L. Boissieux, G. Kiss, N. M. Thalmann, and P. Kalra. "Simulation of skin aging and wrinkles with cosmetics insight", Proc. Eurographics Workshop on ComputerAnimation and Simulation 2000, pp. 15-27, 2000.

4. S. Hadap, E. Bangerter, P. Volino and N. M. Thalmann. "Animating wrinkles on clothes", Proc. IEEE Visualization'99, pp. 175-182, 1999.

5. G. Miller. "The motion dynamics of snakes and worms", Proc. SIGGRAPH'88, vol.22, pp. 169-178, 1988.

6. M. Nahas, H. Huitric, M. Rioux, and J. Domey, "Facial image synthesis using skin texture recording", The Visual Computer, 6(6): 337-343, 1990.

7. D. Terzopoulus and K. Waters. "Physically-based facial modeling, analysis and animation", Journal of Visualization and Computer Animation, vol.1, pp. 73-80, 1990.

8. M. L. Viaud and H. Yahia. "Facial animation with wrinkles", Proc. Eurographics Workshop on Animation and Simulation'92, pp. 1-13, 1992.

9. P. Volino and N. M. Thalmann. "Fast geometrical wrinkles on animated surfaces", Proc. WSCG'99, 1999.

10. K. Waters. "A muscle model for animating three-dimensional facial expression", Proc. SIGGRAPH'87, vol.21, pp. 17-24, July 1987.

11. Y. Wu, P. Kalra, L. Moccozet, and N. M. Thalmann. "Simulating wrinkles and skin aging", The Visual Computer, 15(4): 183-198, 1999.

12. Y. Wu, P. Kalra, and N. M. Thalmann. "Physically-based wrinkle simulation \& skin rendering”, Proc. Eurographics Workshop on Animation and Simulation'97, pp. 69-79, 1997.

13. Y. Zhang, E. C. Prakash, and E. Sung. "Efficient modeling of an anatomy-based face and fast 3D facial expression synthesis." Computer Graphics Forum, 22(2): 159-169, June 2003. 